\title{
Methods for preparing polymer-decorated single exchange-biased magnetic nanoparticles for application in flexible polymer-based films
}

\author{
Laurence Ourry $\ddagger$, Delphine Toulemon ${ }^{\ddagger}$, Souad Ammar ${ }^{*}, \ddagger$ and Fayna Mammeri ${ }^{*}, \neq$
}

\section{Full Research Paper}

\section{Address:}

Université Paris Diderot, Sorbonne Paris Cité, CNRS UMR 7086

ITODYS, Case 7090, 5 rue Thomas Mann, Paris, France

\section{Email:}

Souad Ammar* - ammarmer@univ-paris-diderot.fr; Fayna Mammeri* -

fayna.mammeri@univ-paris-diderot.fr

* Corresponding author $\ddagger$ Equal contributors

\section{Keywords:}

assembly; ATRP; magnetic nanoparticle; exchange-bias; films:

functionalization; polymerization; poly(methyl methacrylate);

polystyrene; seed-mediated growth; surface
Beilstein J. Nanotechnol. 2017, 8, 408-417.

doi:10.3762/bjnano.8.43

Received: 12 October 2016

Accepted: 19 January 2017

Published: 09 February 2017

This article is part of the Thematic Series "Hybrid nanomaterials: from the laboratory to the market".

Guest Editor: A. Taubert

(c) 2017 Ourry et al.; licensee Beilstein-Institut.

License and terms: see end of document.

\begin{abstract}
Background: Magnetic nanoparticles (NPs) must not only be well-defined in composition, shape and size to exhibit the desired properties (e.g., exchange-bias for thermal stability of the magnetization) but also judiciously functionalized to ensure their stability in air and their compatibility with a polymer matrix, in order to avoid aggregation which may seriously affect their physical properties. Dipolar interactions between NPs too close to each other favour a collective magnetic glass state with lower magnetization and coercivity because of inhomogeneous and frustrated macrospin cluster freezing. Consequently, tailoring chemically (through surface functionalization) and magnetically stable NPs for technological applications is of primary importance.
\end{abstract}

Results: In this work, well-characterized exchange-biased perfectly epitaxial $\mathrm{Co}_{x} \mathrm{Fe}_{3-x} \mathrm{O}_{4} @ \mathrm{CoO}$ core@shell NPs, which were isotropic in shape and of about $10 \mathrm{~nm}$ in diameter, were decorated by two different polymers, poly(methyl methacrylate) (PMMA) or polystyrene (PS), using radical-controlled polymerization under various processing conditions. We compared the influence of the synthesis parameters on the structural and microstructural properties of the resulting hybrid systems, with special emphasis on significantly reducing their mutual magnetic attraction. For this, we followed two routes: the first one consists of the direct grafting of bromopropionyl ester groups at the surface of the NPs, which were previously recovered and redispersed in a suitable solvent. The second route deals with an "all in solution" process, based on the decoration of NPs by oleic acid followed by ligand exchange with the desired bromopropionyl ester groups. We then built various assemblies of NPs directly on a substrate or suspended in PMMA.

Conclusion: The alternative two-step strategy leads to better dispersed polymer-decorated magnetic particles, and the resulting nanohybrids can be considered as valuable building blocks for flexible, magnetic polymer-based devices. 


\section{Introduction}

Polymer-based hybrid materials are opening the way for engineering new, multifunctional, flexible materials exhibiting novel properties (e.g., mechanical, magnetic, electrical, optical) due to the synergy between the two components, polymer and inorganic nanoparticles (NPs) [1]. In the case of magnetic hybrids, one of the main challenges is to avoid NP aggregation. The magnetic response of NPs to external magnetic stimulus depends strongly on their intrinsic properties (composition, size, and shape) but also on their spatial arrangement (self-assembly, dispersion in organic media, compatibility with polymers) $[2,3]$ If the interparticle distances are decreased too much, a collective magnetic glass state is set up, reducing magnetization and coercivity because of inhomogeneous and frustrated macrospin cluster freezing [4-6]. Ideally, flexible magnetic devices require dense but well-separated magnetic NPs to decrease interparticle interactions, particularly dipolar ones $[7,8]$.

The general strategy for such a purpose consists of forming core-shell hybrid structures in which the shell consists of a corona of polymer chains grafted onto the inorganic NP surface. Among the available polymer grafting processes, living-radical polymerization (e.g., atom-transfer radical polymerization (ATRP), reversible addition-fragmentation chain transfer (RAFT) or nitroxide-mediated polymerization (NMP)) makes it possible to establish robust polymer-particle bonds and then grow polymer brushes of controlled molecular weight and polydispersity with a satisfactory grafting density. The polymer chains also stabilize the inorganic NPs with respect to the ambient atmosphere and provide compatibility with the resulting polymer matrix.

This strategy has been widely investigated with magnetic NPs. To date, most NPs studied were of iron oxide [9-14]. Exchangebiased NPs (ENPs) have been scarcely considered [15] despite their improved magnetic properties. These particles consist of ferro- or ferrimagnetic (F) cores coated with nanocrystalline antiferromagnetic (AF) layers, and exhibit exchange coupling at the F-AF interface (see for instance [16-18]), leading to an enhanced effective magnetic anisotropy constant $\left(K_{\text {eff }}\right)$ and a higher temperature of transition from a magnetically blocked state to a superparamagnetic one $\left(T_{\mathrm{B}}\right)[19,20]$. Focusing on such particles, in this work, we propose various material processing routes to prepare weakly interacting and densely arranged hybrid ENPs. The ENPs used were prepared by seed-mediated growth in a polyol medium; they consist of ferrimagnetic $\mathrm{Co}_{x} \mathrm{Fe}_{3-x} \mathrm{O}_{4}$ single crystals, almost isotropic in shape and of about $10 \mathrm{~nm}$ in diameter, coated in a perfectly epitaxial fashion with an antiferromagnetic $\mathrm{CoO}$ polycrystalline shell about $1 \mathrm{~nm}$ thick, as described in previous work [16]. We then controlled their surface functionality to tentatively design well-tailored nano-building-blocks for the aforementioned devices. To reduce mutual magnetic attraction and aggregation as far as possible in the first stage of polymer grafting, mechanical stirring and dilute suspensions of reactants were used, even if the functionalization of large amounts of particles becomes difficult. We specifically graft poly(methyl methacrylate) (PMMA) and polystyrene (PS) chains around $\mathrm{Co}_{x} \mathrm{Fe}_{3-x} \mathrm{O}_{4} @ \mathrm{CoO}$. Several key parameters have to be taken into account to realize a controlled polymerization reaction and especially when one aims to graft an ATRP initiator at the surface of particles: the nature of the surface (e.g., oxide or metal) and the interface between the components of the resulting hybrid, namely polymer chains and inorganic NPs (e.g., covalent, ionic, van der Waals). Several halogenated coupling agents can be used to covalently graft an organic group onto the surface of oxide NPs, e.g., organosilanes [21-23], carboxylate [24,25] or phosphonate/phosphate molecules [26,27].

Organosilanes present the disadvantage of condensing after hydrolysis and leading to a thin shell of polysiloxane whose structure and thickness cannot be well controlled. Carboxylates can be degrafted in solution [28], and are, consequently, not the best candidates for the desired applications. Phosphates and phosphonates are suitable for functionalizing iron and silicon oxide surfaces $[29,30]$ through covalent bonds, in mono-, bi- or tridentate modes; but to date, they have not been used for cobalt oxide and ferrite surfaces.

In the case of PMMA, we followed two routes: the first one consists of a direct grafting of bromopropionyl ester molecules ob the surface of ENPs that were previously recovered and redispersed in a suitable solvent. The second uses an "all in solution" process, based on a prior decoration of ENPs by oleic acid ligands, which were subsequently exchanged by the desired bromopropionyl ester species. In the case of PS, we followed exclusively the first route, but in all the cases, the polymer chains were grown by ATRP (see the general synthesis scheme summarized in Figure 1), acting on the NP/monomer weight ratio and on the polymerization time parameters. The resulting nanohybrids were then characterized with special emphasis on the effect of the reaction parameters on their main microstructural properties and taking into account the fact that PS polymerizes more slowly than PMMA.

\section{Results and Discussion}

We chose to work on two very common thermoplastic polymers: PMMA and PS, which have very similar properties (e.g., specific temperatures, mechanical properties, density) but rather different side chains; PMMA has an aliphatic ester group while PS has a more rigid aromatic ring. However, despite their struc- 


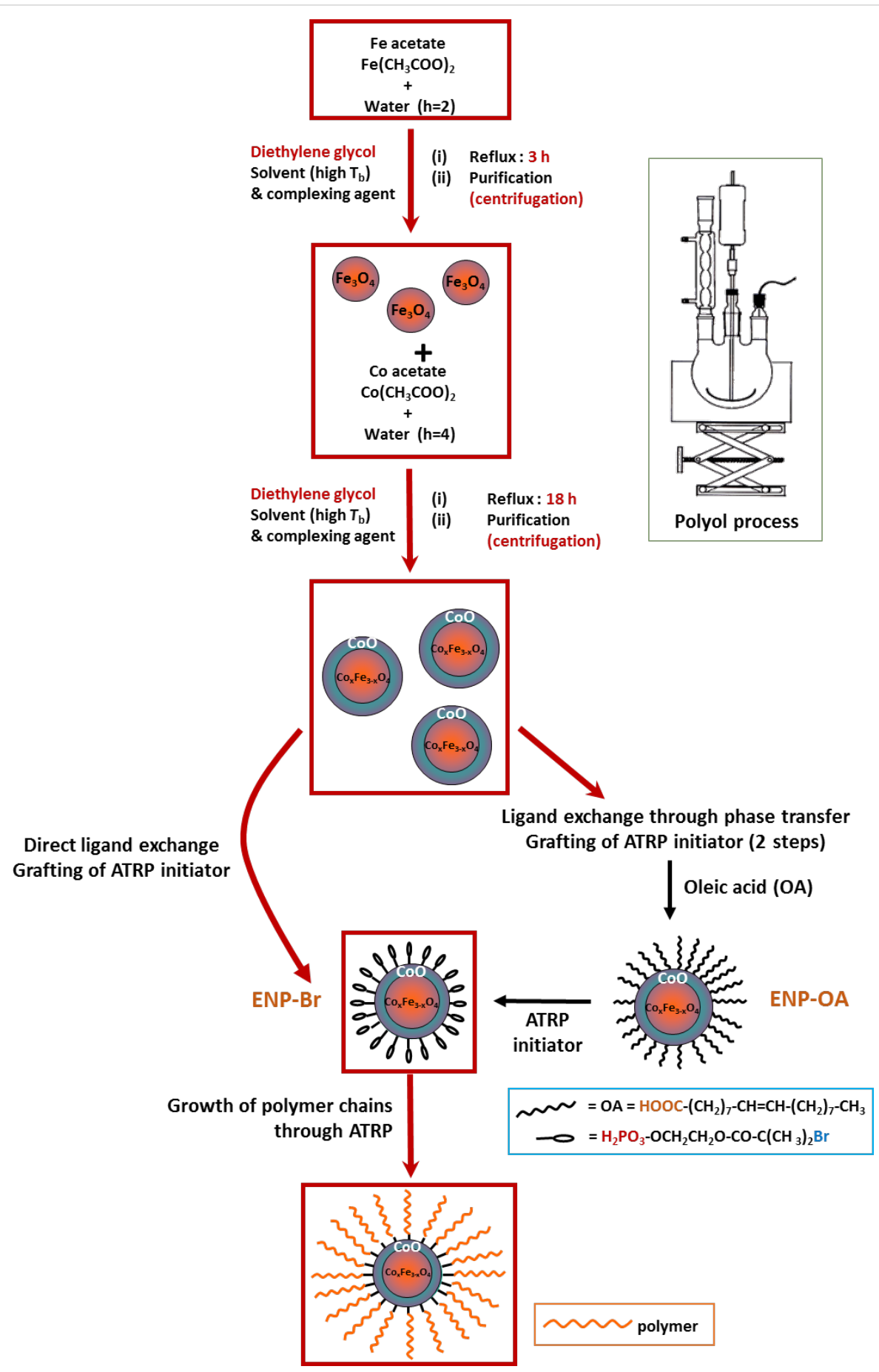

Figure 1: Polymer-decorated exchange-biased $\mathrm{Co}_{x} \mathrm{Fe}_{3-x} \mathrm{O}_{4} @ \mathrm{CoO}$ magnetic nanoparticles synthesis.

tural and reactivity differences, we followed the same reaction pathway to elaborate ENPs decorated by the two polymers (Figure 1).

\section{Growth of PMMA chains}

We previously developed a two-step pathway to produce the hybrid polymer-decorated ENPs [15]. First, an ATRP initiator, 2-phosphonooxy-2-bromo-2-methylpropanoate, was grafted onto the ENP surface. Under the conditions used (see Experimental), the grafting density of the initiator molecules is 2.8 molecules $\mathrm{nm}^{-2}$, in good agreement with previous studies [27]. Then, the PMMA chains were grown by ATRP at $30^{\circ} \mathrm{C}$ in the presence of cuprous bromide and $N, N, N^{\prime}, N^{\prime}, N^{\prime \prime}$ pentamethyldiethylenetriamine (PMDTA) to form the 
catalytic $\mathrm{Cu}$-PMDETA complex, with polymerization times of 1 to $3 \mathrm{~h}$. The polymer coating was characterized by X-ray photoelectron spectroscopy (XPS) and thermogravimetric analysis (TGA) [15]. For all samples the grafted chain density was 1.4 chain $\mathrm{nm}^{-2}$, i.e., significantly higher than the results previously reported [15]. A chain density ranging between 0.1 and 1 chain $\mathrm{nm}^{-2}$ is commonly reported $[13,26,31,32]$. In the present case, a higher grafting density means better protection of the magnetic particles against oxidation and more stable magnetic properties over time [33]. Indeed, the aim here is not necessarily to grow very long polymer chains which are diamagnetic, but to functionalize ENPs efficiently in order to increase their compatibility with polymer matrices. Comparison of the grafting densities obtained here with those reported elsewhere suggests that, for ENPs of similar size, phosphates and phosphonates are better grafted and in greater quantity to the surface of ENPs than carboxylates and organosilanes.

\section{Growth of PS chains}

Styrene polymerizes more slowly than methyl methacrylate. Yousi et al. [34] demonstrated that the propagation rate, for similar conversion yields, can be increased by catalysts. Masson et al. [35] reported an increase in the styrene polymerization rate, using malonitrile as a catalyst and from initiator molecules anchored on iron oxide NPs. However, it appears that bonding the initiator to the surface through a phosphonate group limits the rate, reducing the effect of the catalyst. The length of the carbon backbone of the initiator (between phosphate and $\alpha$-bromo-ester functions) is very important to the ATRP polymerization rate when initiators are directly anchored to the particle surface. Sunday et al. [36] reported that long alkyl chains (16 carbon atoms) or short ones (3 carbon atoms) lead to higher polymerization rates and grafting densities than intermediate chains (e.g., 11 carbon atoms). Masson et al. used an 11-carbonlong initiator. That which we used, the same as for PMMA (2-phosphonooxy-2-bromo-2-methylpropanoate), has only two carbon atoms, and might be expected to give good polymerization rates.

Working under the same conditions previously used for PMMA growth on ENPs, we prepared a series of samples by varying the polymerization time from 18 to $24 \mathrm{~h}$; the resulting hybrids will be referred to as ENP-PS-h (where h corresponds to the polymerization time in hours). The survey XPS spectra of the resulting hybrids evidence all the characteristic signals of ENPs and initiator species (Figure 2). Hence, the O 1s (531.0 eV), Fe 2 $\mathrm{p}_{3 / 2}(711.5 \mathrm{eV})$, Co $2 \mathrm{p}_{3 / 2}(781.7 \mathrm{eV}), \mathrm{P} 2 \mathrm{p}$ $(133.5 \mathrm{eV})$, and $\mathrm{Br} 3 \mathrm{p}_{3 / 2}(190.7 \mathrm{eV})$ peaks can be assigned to $\mathrm{Co}_{x} \mathrm{Fe}_{3-x} \mathrm{O}_{4} @ \mathrm{CoO}$ and $\mathrm{PO}(\mathrm{OH})_{2} \mathrm{O}\left(\mathrm{CH}_{2}\right)_{2} \mathrm{OCOC}\left(\mathrm{CH}_{3}\right)_{2} \mathrm{Br}$ phases, respectively. The absence of $\mathrm{Cu}$ signals in the ENP-PS spectra suggests that all metallic $\mathrm{Cu}$ complexes were eliminated during the washing and purification steps. Chemical compositions (provided by XPS data) of as-produced NPs and ENP-PS are listed in Table 1.

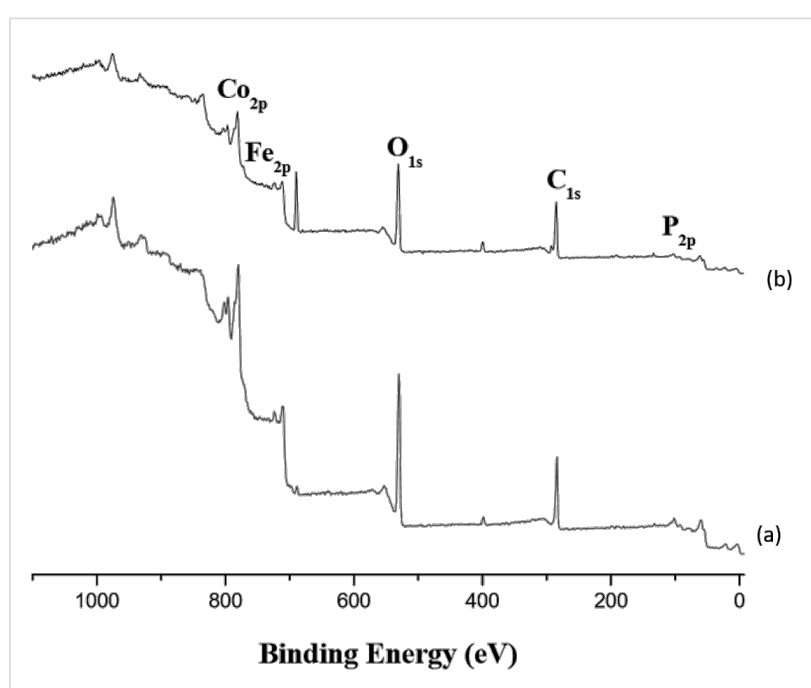

Figure 2: XPS survey spectra of (a) ENP-PS-18, (b) ENP-PS-24.

Table 1: XPS-determined atomic composition of as-prepared $\mathrm{Co}_{x} \mathrm{Fe}_{3-x} \mathrm{O}_{4}-\mathrm{CoO}$ nanoparticles (ENP) and PS-based hybrids.

\begin{tabular}{lcccccc} 
& \multicolumn{6}{c}{ Elemental atomic composition (\%) } \\
& $\mathrm{Fe}$ & $\mathrm{Co}$ & $\mathrm{O}$ & $\mathrm{C}$ & $\mathrm{P}$ & $\mathrm{Br}$ \\
& & & & & & \\
\hline ENP & 14.5 & 24.3 & 40.2 & 21.0 & - & - \\
$\left(\mathrm{Co}_{x} \mathrm{Fe}_{3-x} \mathrm{O}_{4} @ \mathrm{CoO}\right)^{*}$ & & & & & & \\
ENP-PS-18 & 7.7 & 10.5 & 37.6 & 41.5 & 2.3 & 0.4 \\
ENP-PS-24 & 7.4 & 11.6 & 37.7 & 41.8 & 1.2 & 0.3 \\
\hline
\end{tabular}

*Reproduced in part with permission from [15]. Copyright 2016 The Royal Society of Chemistry.

The C 1s peak prior to functionalization indicates that organic residues (polyol and acetate molecules) are present at the core-shell NP surface. The Co $2 p$ and Fe $2 p$ peaks are much weaker after polymerization due the presence of a significant polymer coating. The decomposition of the $\mathrm{C} 1 \mathrm{~s}$ peak for polystyrene (PS) was difficult since there are many sources of organic matter (PS, initiator, residual polyol and acetates); however, unfortunately, styrene contains no other element suitable for XPS analysis. However, IR spectra of PS-decorated ENPs (Figure 3) exhibit several peaks characteristic of polystyrene: $3024 \mathrm{~cm}^{-1}\left(v_{\mathrm{as}\left(\mathrm{CH} 2 \_a r o m\right.}\right), 2950 \mathrm{~cm}^{-1}\left(v_{\mathrm{s}\left(\mathrm{CH} \_ \text {aliph }\right)}\right)$ and $1600 \mathrm{~cm}^{-1}\left(v_{\mathrm{C}=\mathrm{C}}\right)$ when compared to a commercial reference.

Similar to the PMMA-based nanohybrids, the amount of cobalt was found to be higher than that of iron for PS-functionalized ENPs. The mean free paths of $1.5 \mathrm{~nm}$ for iron in $\mathrm{CoO}$ and $1.4 \mathrm{~nm}$ for cobalt in $\mathrm{CoO}$ were calculated following the method 


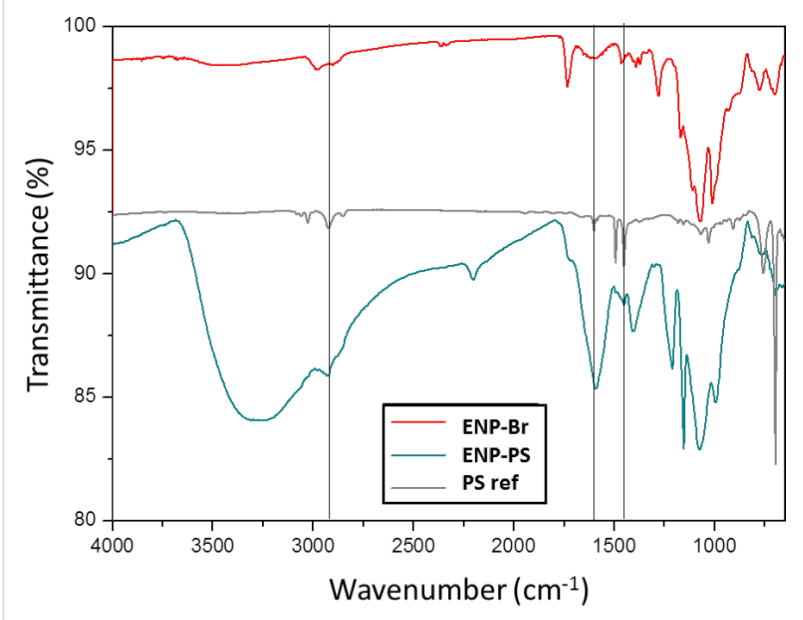

Figure 3: IR spectrum of ENP-PS-18 and those of free PS and ENPS functionalized by the ATRP initiator only (ENP-Br).

described previously [15]. However, electrons pass only through $0.5 \mathrm{~nm}$ of the Fe-rich core after having penetrated the $1 \mathrm{~nm}$ thick $\mathrm{CoO}$ shell, leading to a difference in depth analysis of the core and the shell. Then, we assumed that cobalt and carbon are representative of the $\mathrm{CoO}$ shell [30] and the PS brushes, respectively, to determine the PS thickness from the $\mathrm{C} 1 \mathrm{~s}$ and Co $2 p$ peaks and their relative intensities. The thicknesses were found to be $2.5 \pm 0.5 \mathrm{~nm}$ for ENP-PS-18 and $3.0 \pm 0.5 \mathrm{~nm}$ for ENP-PS-24. However, in the XPS chamber (under ultravacuum conditions), the polymer chains tend to collapse. Therefore, one must consider that XPS measurements do not allow the exact chain length to be determined but that of a polymer in a random coil conformation. Nevertheless, we can conclude that more polystyrene is present around the ENP when the polymerization time increases. TGA thermograms of PS-decorated ENPs are presented in Figure 4. PS is generally decomposed at about $380{ }^{\circ} \mathrm{C}$. It can be seen that, although the polymer is thicker at the ENP surface in the case of PS, the weight losses of PS (6\% and $14 \%$ for NP-PS-18 and NP-PS-24, respectively) are lower

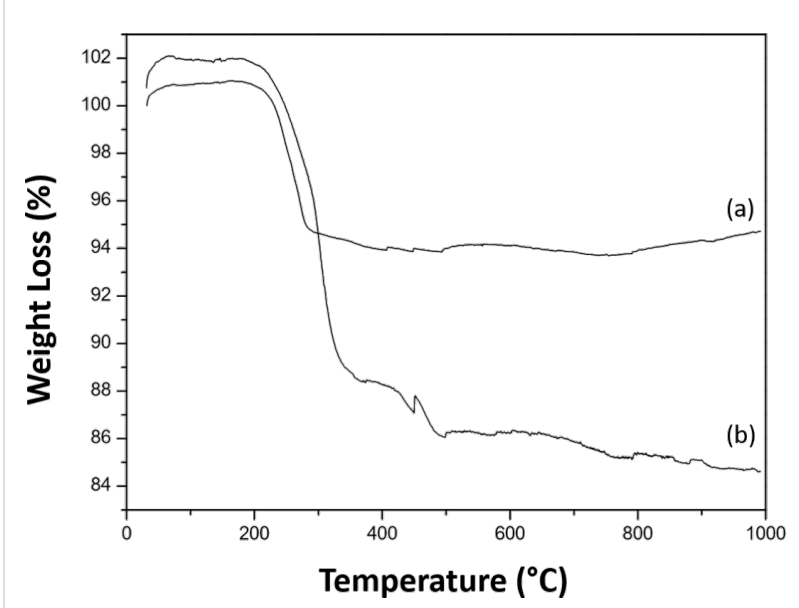

Figure 4: TGA curves of (a) ENP-PS-18 and (b) ENP-PS-20.

than those of PMMA in ENP-PMMA-1 and ENP-PMMA-3 [15], meaning that PS collapses less than PMMA. This is doubtful due to the presence of aromatic rings.

TEM images presented in Figure 5 depict well-dispersed nanoparticles, although the surface of the grid was not totally covered with ENPs. Similar images have been obtained for the two samples. There are spaces between the ENPs that are close to each other, suggesting that they are separated by PS coatings.

\section{Ligand exchange for improved separation of magnetic NPs}

In the previous sections, we demonstrated the feasibility of preparing polymer (PMMA or PS) functionalized magnetic particles with improved polymer chain grafting densities than those previously reported. However, it is still a serious challenge to separate all the particles and avoid a few aggregates. Hence, we replaced the centrifugation of the ENPs after the polyol synthesis by direct ligand exchange between adsorbed polyol molecules and oleic acid (OA) in the reaction mixture (see Experi-
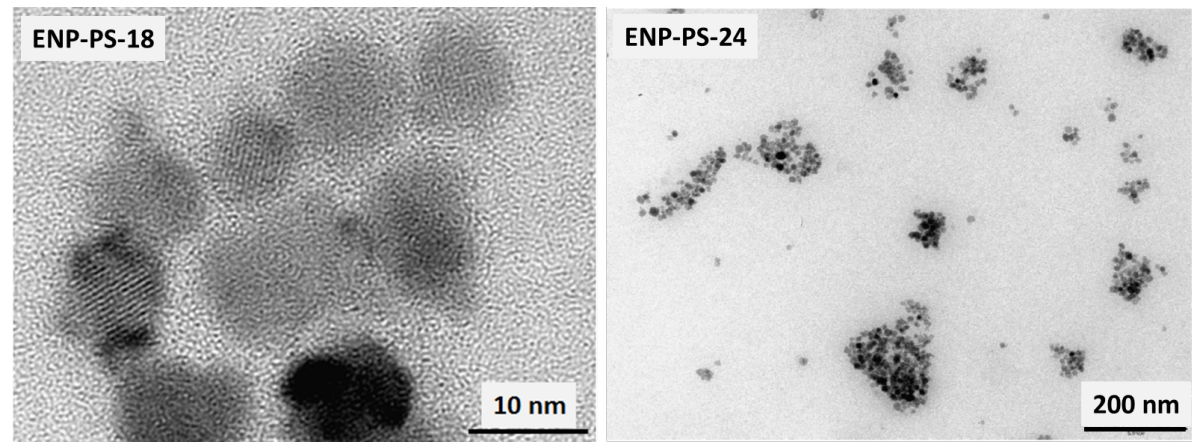

Figure 5: TEM images of ENP-PS-18 (left) and ENP-PS-24 (right) 
mental). Oleic acid is known to cap oxide nanoparticles by ionic bonding $[24,25]$. Moreover, it bears a double bond $\mathrm{C}=\mathrm{C}$, inducing a degree of structural rigidity and a kink in the chain, promoting the spacing of the particles during their organization. TEM images (Figure 6) clearly show a better separation of the ENPs previously coated with OA instead of being recovered simply by centrifugation of the polyol mixture.

Figure 7 shows the variation of the size distribution of the magnetic particles as a function of the surface state (with or without oleic acid), determined by dynamic light scattering (DLS) measurements. The distribution is found to be quite polydisperse when ENPs are purified by centrifugation from the polyol and narrower when they are coated with OA. Moreover, the average diameter is found to be $\approx 100 \mathrm{~nm}$ for the former and $25 \mathrm{~nm}$ for the latter, which shows that the functionalization by OA through ligand exchange leads to a better separation of exchange-biased magnetic particles. Then, PMMA chains were grown from the surface of ENP-Br by the experimental procedure described previously [15].

Figure 8 presents the TEM images. Better-separated ENPs are recovered when oleic acid is used, and they are, consequently, more adapted for applications in flexible polymer devices.

\section{Assembly of PMMA-decorated magnetic nanoparticles}

Finally, we assembled PMMA-functionalized ENPs prepared by the ligand exchange procedure and dispersed in THF. Thin films were prepared by drop casting on silicon wafers and SEM images were recorded. Interestingly, Figure 9 depicts an incomplete monolayer of ENPs (with a scheme presented in the insert).

Then, ENP-PMMA was introduced in a solution of PMMA in THF, where the ENP/PMMA ratio was varied from 1:1 to 1:9. Large aggregates were obtained at higher concentrations of

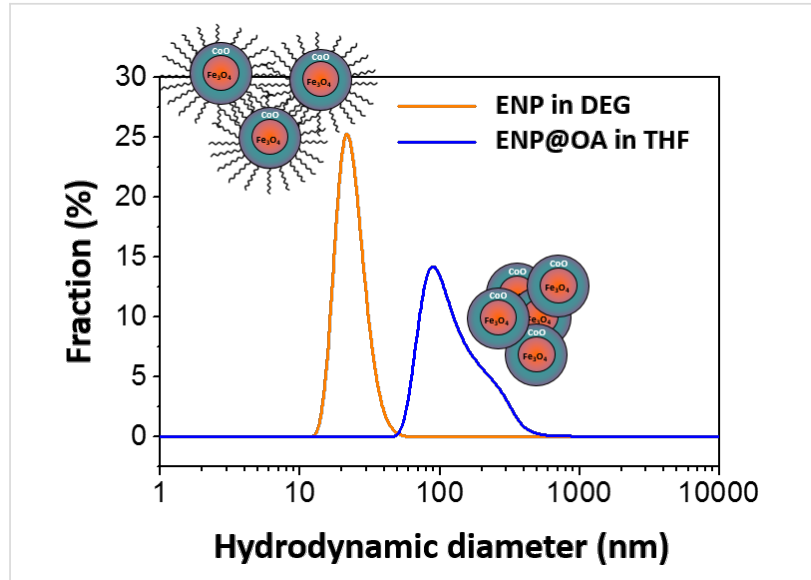

Figure 7: Size distributions of ENPs, in polyol solvent (diethylene glycol) and functionalized by oleic acid, dispersed in tetrahydrofuran solvent (THF), obtained by DLS.

ENPs and small aggregates or isolated ENPs at higher dilutions. SEM images (Figure 10) present well-dispersed ENPs in both cases, suggesting that the ENP-PMMA NPs are very well separated and can be dispersed and assembled in a controlled manner, thanks to the polymer grafted on the ENP surface.

The production of such nanoparticles thus opens the way to various applications such as: (i) the fundamental study of the magnetic properties of the various assemblies of these magnetic ENPs. There is still intensive research to be done on the control of the magnetic properties of magnetic films, either comprised of nanocomposites or not [37], via the spatial ENP orientation $[28,38]$ to be combined with polymer processing $[39,40]$.

As a preliminary result, focusing on these PMMA-decorated ENP assemblies, a net decrease of the blocking temperature value, defined as the critical temperature at the relaxed/blocked magnetic states transition, was observed when the ENP dilution ratio was increased (Figure 11). Such behaviour is
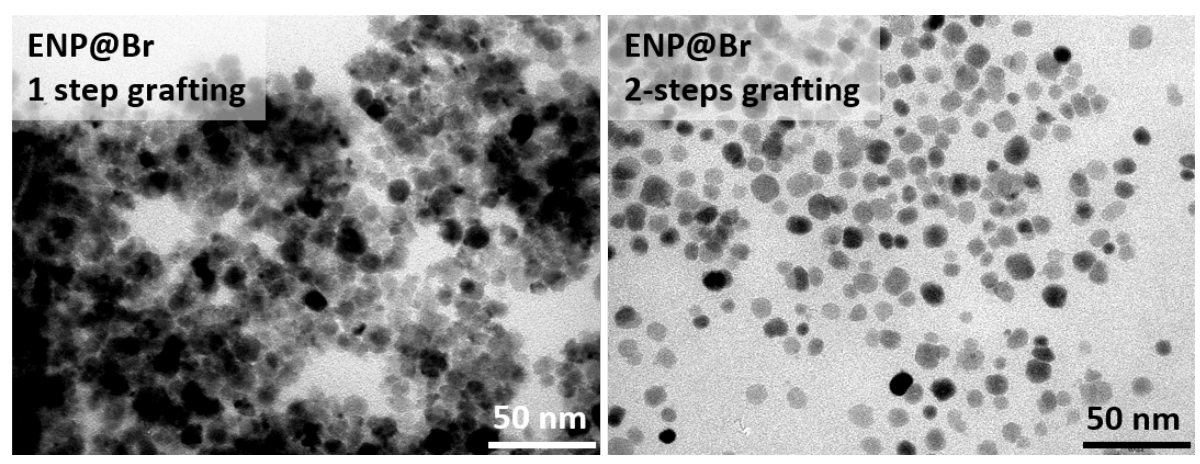

Figure 6: TEM images of a) ENP-Br obtained after centrifugation of as-prepared ENPs and direct grafting of ATRP initiator; b) ENP-Br obtained by two-step ligand exchange by oleic acid followed by grafting of ATRP initiator. 

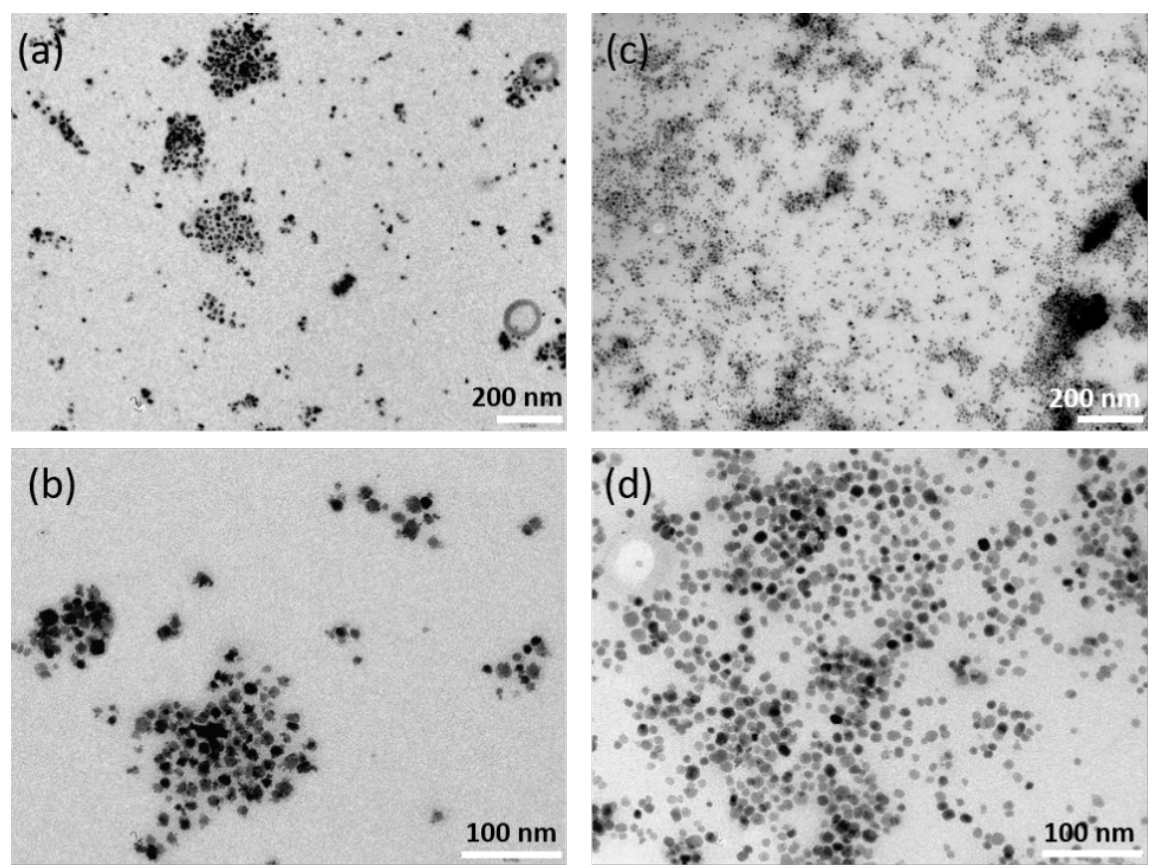

Figure 8: TEM images of magnetic ENPs decorated with PMMA brushes: $(a, b)$ ENP-Br obtained through direct grafting of ATRP initiator after $3 \mathrm{~h}$ polymerization; (c,d) ENP-Br obtained by ligand exchange (oleic acid/ATRP initiator) under the same conditions.

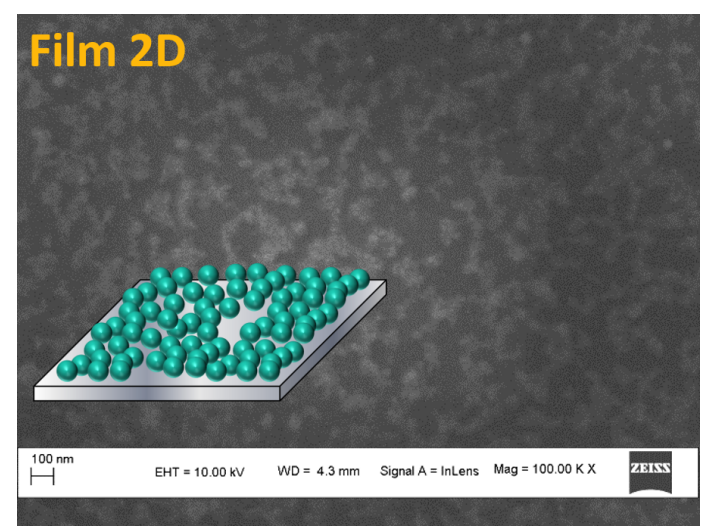

Figure 9: Thin films prepared by drop casting of a suspension of ENPPMMA in THF. quite common for superparamagnetic single-domain nanoparticles, and it is here respected in the case of exchange-biased nanoparticles.

It is clear that the material processing approach we proposed here, namely, the controlled surface polymerization of oxidebased ENPs, is able to tune their magnetic properties. Further magnetic measurements are still in progress to fully appreciate the dipolar interaction effect on the exchange bias and the influence of NP assembly on the exchange field.

\section{Conclusion}

We described the functionalization of $10 \mathrm{~nm}$ exchange-biased $\mathrm{Co}_{x} \mathrm{Fe}_{3-x} \mathrm{O}_{4} @ \mathrm{CoO}$ core@shell NPs by two different polymers, poly(methyl methacrylate) (PMMA) and polystyrene (PS),
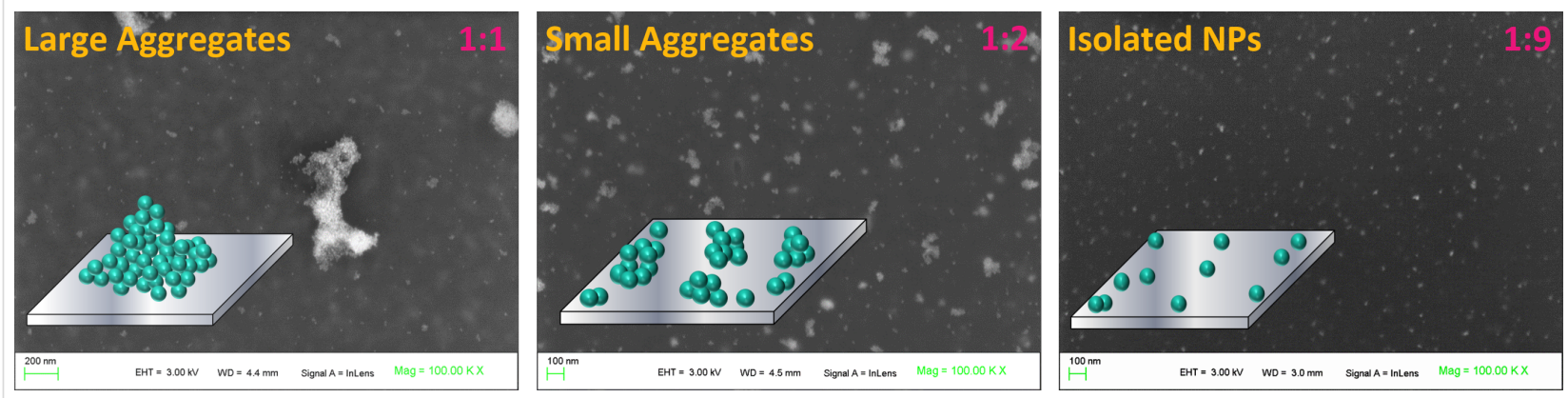

Figure 10: SEM images of various assemblies of ENP-PMMA, obtained by drop casting from THF suspension with ENP/PMMA ratios of 1:1, 1:2 and $1: 9$. 


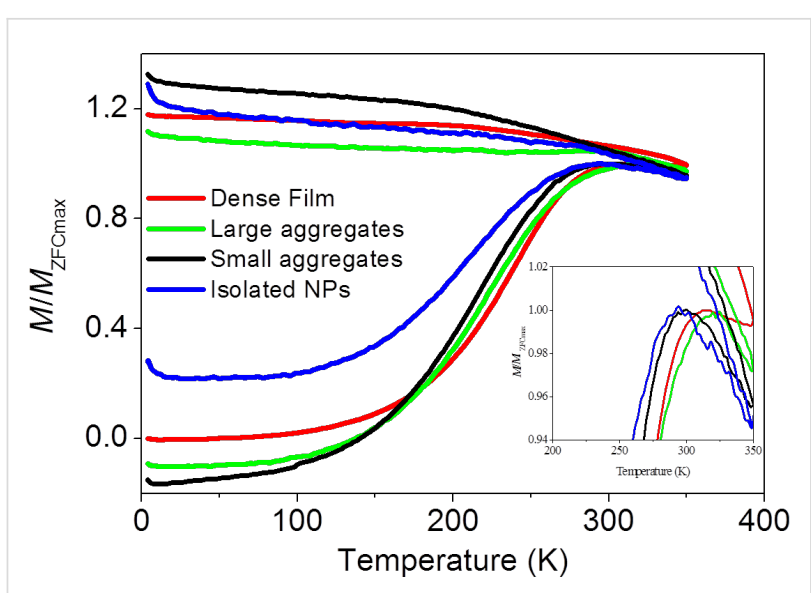

Figure 11: Thermal variation of the normalized dc magnetic magnetization measured in zero-field cooling (ZFC) conditions for the assembled PMMA-decorated ENP sample series (see Figure 10). Details around the maximum of the magnetization is given in the inset.

using radical-controlled polymerization under various processing conditions. We evidenced through TGA, IR spectroscopy and XPS measurements that polymer chains were efficiently grafted onto the nanoparticles using either the direct grafting or the "all in solution" process. In the TEM images we have also observed that very little aggregation occurs. Nevertheless, the alternative two-step strategy leads to better dispersed polymerdecorated magnetic particles, and the resulting nanohybrids can be considered as valuable building blocks for flexible, magnetic polymer-based devices. We also developed various assemblies by varying the dilution of the ENP suspension in THF, where the assemblies varied from small aggregates to isolated ENPs at the surface of silicon substrates. These ENPs allow for the preparation of flexible, functional, hybrid PMMA-ENP films with enhanced properties (e.g., magnetic, mechanical)

\section{Experimental}

\section{Hybrid synthesis}

PMMA chain growth by ATRP (direct ligand exchange for ATRP initiator grafting) was used as described in [15]: ENPs and 2-phosphonooxy-2-bromo-2-methylpropanoate were introduced in THF and sonicated at room temperature (RT). The resulting nanohybrids were then dispersed in acetonitrile before adding $N, N, N^{\prime}, N^{\prime}, N^{\prime \prime}$-pentamethyldiethylenetriamine (PMDTA) methyl methacrylate. The mixture was degassed with argon and mechanically stirred for $1 \mathrm{~h}$. CuBr was added and the solution was heated around $30^{\circ} \mathrm{C}$ and sonicated under inert atmosphere. The polymerization time was tuned from 1 to $3 \mathrm{~h}$ to influence the length of the polymer chains. The reaction was quenched by opening the system and diluting the solution with THF and hexane. Hairy, hybrid ENPs were recovered by centrifugation and washing with THF. They were referred to as ENP-PMMA$\mathrm{h}$, where $\mathrm{h}$ corresponds to the polymerization time in hours.
PS chain growth (direct ligand exchange for ATRP initiator grafting) proceeded by first dispersing $80 \mathrm{mg}$ of ENP-Br in $37 \mathrm{~mL}$ of toluene before adding $8.2 \mu \mathrm{L}$ of PMDTA, $4.8 \mathrm{~mL}$ of styrene and $11.4 \mathrm{~mL}$ of malonitrile in order to arrive at the ratio styene/initiator/malonitrile/PMDETA/CuBr 1000:1:4:1:1. The mixture was degassed with argon and mechanically stirred for at least 1 hour. $5.7 \mathrm{mg}$ of $\mathrm{CuBr}$ were added and the solution was heated at $90{ }^{\circ} \mathrm{C}$ and mechanically stirred under argon. The polymerization time was varied from 18 to $24 \mathrm{~h}$. Finally, the reaction was quenched by opening the system to the atmosphere. The resulting hybrids were referred to as ENP-PS-h, where $h$ corresponds to the polymerization time in hours.

PMMA chain growth (two-step ATRP initiator grafting, ligand exchange by phase transfer) was accomplished using $150 \mathrm{mg}$ of ENPs, dispersed in $45 \mathrm{~mL}$ of diethylene glycol, which was added to $75 \mathrm{~mL}$ of a solution of oleic acid in toluene $(10 \% \mathrm{v} / \mathrm{v})$ and sonicated for $15 \mathrm{~min}$ and left overnight. The OA-functionalized ENPs were recovered from the toluene layer and $375 \mathrm{mg}$ of 2-phosphonooxy-2-bromo-2-methylpropanoate were added to the suspension and sonicated for $24 \mathrm{~h}$. Toluene was then removed and the functionalized ENPs were recovered after several washings with THF.

\section{Hybrid characterization}

The different reaction steps were monitored by ATR-FTIR on a Thermo Nicolet 8700 spectrometer equipped with a diamond crystal (50 scans, $4 \mathrm{~cm}^{-1}$ resolution). Thermogravimetric analyses (TGA) were performed in air on a Labsys-Evo device with a heating rate of $10{ }^{\circ} \mathrm{C} \mathrm{min}^{-1}$. X-ray photoelectron spectroscopy (XPS) measurements were performed on as-prepared and functionalized ENPs using a Thermo VG ESCALAB 250 instrument equipped with a micro-focused, monochromatic Al $\mathrm{K} \alpha \mathrm{X}$-ray source $(1486.6 \mathrm{eV})$ and a magnetic lens. The X-ray spot size was $500 \mu \mathrm{m}(15 \mathrm{kV}, 150 \mathrm{~W})$. The spectra were acquired in the constant analyser energy mode with pass energies of 150 and $40 \mathrm{eV}$ for the general survey and the narrow scans, respectively. The samples were fixed on sample holders and out-gassed in the fast entry airlock $\left(2 \times 10^{-7} \mathrm{mbar}\right)$. The Avantage software package was used for data acquisition and processing. The $\mathrm{C} 1 \mathrm{~s}$ line of $285 \mathrm{eV}$ was used as the reference to correct the binding energies. Scanning electron microscopy (SEM) and transmission electron microscopy (TEM) were performed on Supra40 ZEISS FEG-SEM and JEOL-100-CX II TEM microscopes, operating at 5.0 and $100 \mathrm{kV}$, respectively.

\section{Acknowledgements}

The authors would like to thank Dr. Thomas Gaudisson (who designed the $\mathrm{Co}_{x} \mathrm{Fe}_{3-x} \mathrm{O}_{4} @$ CoO ENPs) and Dr. John Lomas for fruitful discussions, Ms. Smina Hammaz for her technical support on polymer brush degrafting, Dr. Philippe Decorse for 
the XPS measurements and M. Ludovic Mouton for recording the TEM images.

The ANR (Agence Nationale de la Recherche) and CGI (Commissariat à l'Investissement d'Avenir) are gratefully acknowledged for their financial support of this study through Labex SEAM (Science and Engineering for Advanced Materials and Devices) ANR 11 LABX 086, ANR 11 IDEX 0502.

\section{References}

1. Balazs, A. C.; Emrick, T.; Russel, T. P. Science 2006, 314, 1107-1110. doi:10.1126/science.1130557

2. Frankamp, B. L.; Boal, A. K.; Tuominen, M. T.; Rotello, V. M. J. Am. Chem. Soc. 2005, 127, 9731-9735. doi:10.1021/ja051351m

3. Fleutot, S.; Nealon, G. L.; Pauly, M.; Pichon, B. P.; Leuvrey, C.; Drillon, M.; Gallani, J.-L.; Guillon, D.; Donnio, B.; Begin-Colin, S. Nanoscale 2013, 5, 1507-1516. doi:10.1039/c2nr32117c

4. Dormann, J. L.; Fiorani, D.; Cherkaoui, R.; Spinu, L.; Lucari, F.; D'Orazio, F.; Noguès, M.; Tronc, E.; Jolivet, J. P.; Garcia, A. Nanostruct. Mater. 1999, 12, 757-762. doi:10.1016/S0965-9773(99)00231-7

5. Fiorani, D.; Testa, A. M.; Tronc, E.; Lucari, F.; D'Orazio, D.; Nogués, M. J. Magn. Magn. Mater. 2001, 226-230, 1942-1944. doi:10.1016/S0304-8853(00)00680-6

6. Beji, Z.; Smiri, L. S.; Yaacoub, N.; Grenèche, J.-M.; Menguy, N.; Ammar, S.; Fiévet, F. Chem. Mater. 2010, 22, 1350-1366. doi:10.1021/cm901969c

7. Liu, Y.-W.; Zhan, Q.-F.; Li, R.-W. Chin. Phys. B 2013, 22, 127502. doi:10.1088/1674-1056/22/12/127502

8. Ghoneim, M. T.; Hussain, M. M. Electronics (Basel, Switz.) 2015, 4, 424-479. doi:10.3390/electronics4030424

9. Wang, Y.; Teng, X.; Wang, J.-S.; Yang, H. Nano Lett. 2003, 3, 789-793. doi:10.1021/nl034211o

10. Xu, G.; Wu, W.-T.; Wang, Y.; Pang, W.; Zhu, Q.; Wang, P.; You, Y. Polymer 2006, 47, 5909-5918. doi:10.1016/j.polymer.2006.06.027

11. Ninjbadgar, T.; Yamamoto, S.; Fukuda, T. Solid State Sci. 2004, 6, 879-885. doi:10.1016/j.solidstatesciences.2003.11.009

12. Garcia, I.; Zafeiropoulos, N. E.; Janke, A.; Tercjak, A.; Eceiza, A.; Stamm, M.; Mondragon, I. J. Polym. Sci., Part A: Polym. Chem. 2007, 45, 925-932. doi:10.1002/pola.21854

13. Galeotti, F.; Bertini, F.; Scavia, G.; Bolognesi, A. J. Colloid Interface Sci. 2011, 360, 540-547. doi:10.1016/j.jcis.2011.04.076

14. Basti, H.; Ben Tahar, L.; Smiri, L. S.; Herbst, F.; Nowak, S.; Mangeney, C.; Ammar, S. Colloids Surf., A 2016, 490, 222-231. doi:10.1016/j.colsurfa.2015.11.013

15. Ourry, L.; Mammeri, F.; Toulemon, D.; Gaudisson, T.; Delamar, M.; Ammar, S. RSC Adv. 2016, 6, 49973-49979. doi:10.1039/C6RA06963K

16. Gaudisson, T.; Ourry, L.; Hammoud, H.; Nowak, S.; Menguy, N.; Yaacoub, N.; Grenèche, J.-M.; Mammeri, F.; Ammar, S. J. Nanopart. Res. 2014, 16, 2359. doi:10.1007/s11051-014-2359-5

17. Baaziz, W.; Pichon, B. P.; Lefevre, C.; Ulhaq-Bouillet, C.; Grenèche, J.-M.; Toumi, M.; Mhiri, T.; Bégin-Colin, S. J. Phys. Chem. C 2013, 117, 11436-11447. doi:10.1021/jp402823h

18. Chandra, S.; Biswas, A.; Khurshid, H.; Li, W.; Hadjipanayis, G. C.; Srikanth, H. J. Phys.: Condens. Matter 2013, 25, 426003. doi:10.1088/0953-8984/25/42/426003
19. Weller, D.; Moser, A. IEEE Trans. Magn. 1999, 35, 4423-4439. doi:10.1109/20.809134

20. Fullerton, E. E.; Margulies, D. T.; Schabes, M. E.; Carey, M.; Gurney, B.; Moser, A.; Best, M.; Zeltzer, G.; Rubin, K.; Rosen, H.; Doerner, M. Appl. Phys. Lett. 2000, 77, 3806-3808. doi:10.1063/1.1329868

21. Sun, Y.; Ding, X.; Zheng, Z.; Cheng, X.; Hu, X.; Peng, Y. Eur. Polym. J. 2007, 43, 762-772. doi:10.1016/j.eurpolymj.2006.10.021

22. Ohno, K.; Mori, C.; Akashi, T.; Yoshida, S.; Tago, Y.; Tsujii, Y.; Tabata, Y. Biomacromolecules 2013, 14, 3453-3462. doi:10.1021/bm400770n

23. Huang, Y.; Ishige, R.; Tsujii, Y.; Ohno, K. Langmuir 2015, 31 , 1172-1179. doi:10.1021/la504429c

24. Fan, Q.-L.; Neoh, K.-G.; Kang, E.-T.; Shuter, B.; Wang, S.-C. Biomaterials 2007, 28, 5426-5436. doi:10.1016/j.biomaterials.2007.08.039

25. Gravano, S. M.; Dumas, R.; Liu, K.; Patten, T. E. J. Polym. Sci., Part A: Polym. Chem. 2005, 43, 3675-3688. doi:10.1002/pola.20823

26. Babu, K.; Dhamodharan, R. Nanoscale Res. Lett. 2009, 4, 1090-1102. doi:10.1007/s11671-009-9365-z

27. Babu, K.; Dhamodharan, R. Nanoscale Res. Lett. 2008, 3, 109. doi:10.1007/s11671-008-9121-9

28. Mammeri, F.; Le Bras, Y.; Daou, T. J.; Gallani, J.-L.; Colis, S.; Pourroy, G.; Donnio, B.; Guillon, D.; Bégin-Colin, S. J. Phys. Chem. B 2009, 113, 734-738. doi:10.1021/jp808177y

29. Mutin, P. H.; Guerrero, G.; Vioux, A. Chimie 2003, 6, 1153-1164. doi:10.1016/j.crci.2003.07.006

30. Daou, T. J.; Begin-Colin, S.; Grenèche, J.-M.; Thomas, F.; Derory, A.; Bernhardt, P.; Legaré, I. P.; Pourroy, G. Chem. Mater. 2007, 19, 4494-4505. doi:10.1021/cm071046v

31. Huang, C.; Tassone, T.; Woodberry, K.; Sunday, D.; Green, D. L. Langmuir 2009, 25, 13351-13360. doi:10.1021/la901918v

32. Patil, R. R.; Turgman-Cohen, S.; Šrogl, J.; Kiserow, D.; Genzer, J. ACS Macro Lett. 2015, 4, 251-254. doi:10.1021/mz5007188

33. Sayed Hassan, R.; Gaudisson, T.; Yaacoub, N.; Grenèche, J.-M.; Menguy, N.; Nedelka, N.; Slawaska-Waniewska, A.; Galmiche, M.; Mammeri, F.; Ammar, S. Mater. Res. Express 2014, 1, 025035. doi:10.1088/2053-1591/1/2/025035

34. Jiaming, Z.; Rui, L.; Jianying, H.; Jiayan, C.; Xurong, L.; Yutai, L.; Yousi, Z. J. Polym. Sci., Part A: Polym. Chem. 2007, 45, 4082-4090. doi:10.1002/pola.22217

35. Vergnat, V.; Pourroy, G.; Masson, P. Polym. Int. 2013, 62, 878-883. doi:10.1002/pi.4435

36. Sunday, D.; Curras-Medina, S.; Green, D. L. Macromolecules 2010, 43, 4871-4878. doi:10.1021/ma1004259

37. Lim, G.-H.; Lee, J.; Kwon, N.; Bok, S.; Sim, H.; Moon, K.-S.; Lee, S.-E.; Lim, B. Electron. Mater. Lett. 2016, 12, 574-579. doi:10.1007/s13391-016-6179-x

38. Koo, J.; Kim, H.; Kim, K.-Y.; Jang, Y. R.; Lee, J.-S.; Yoon, S. W.; Suh, B. J.; Yu, T.; Bang, J.; Yoon, K.; Yuan, G.; Satija, S. K. RSC Adv. 2016, 6, 55842-55847. doi:10.1039/C6RA10026K

39. Ourry, L.; Marchesini, S.; Bibani, M.; Mercone, S.; Ammar, S.; Mammeri, F. Phys. Status Solidi A 2015, 212, 252-258. doi:10.1002/pssa.201431563

40. Ben Osman, C.; Nowak, S.; Garcia-Sanchez, A.; Charles, Y.; Ammar, S.; Mercone, S.; Mammeri, F. Eur. Polym. J. 2016, 84, 602-611. doi:10.1016/j.eurpolymj.2016.09.056 


\section{License and Terms}

This is an Open Access article under the terms of the Creative Commons Attribution License

(http://creativecommons.org/licenses/by/4.0), which permits unrestricted use, distribution, and reproduction in any medium, provided the original work is properly cited.

The license is subject to the Beilstein Journal of Nanotechnology terms and conditions:

(http://www.beilstein-journals.org/bjnano)

The definitive version of this article is the electronic one which can be found at:

doi:10.3762/bjnano.8.43 